F. S. Cater, Department of Mathematics, Portland State University, Portland, Oregon 97207, USA

\title{
ON THE DINI DERIVATES OF A PARTICULAR FUNCTION
}

\begin{abstract}
We construct a continuous strictly increasing function such that at each point one of its right Dini derivates is 0 or $\infty$, and at each point one of its left Dini derivates is 0 or $\infty$. Thus at no point can it have a positive real unilateral derivative.
\end{abstract}

In $[1,(18.8)]$ there is discussed a continuous strictly increasing function $F$ (attributed chiefly to Riesz-Nagy) that has no real positive derivative at any point. Consequently $F^{\prime}=0$ almost everywhere.

Put another way, $F$ satisfies the condition:

(*) there are no positive real number $y$ and point $x$ such that

$$
D^{+} F(x)=D_{+} F(x)=D^{-} F(x)=D_{-} F(x)=y
$$

where $D^{+}, D_{+}, D^{-}, D_{-}$denote the usual Dini derivates.

But $F$ may not satisfy the stronger condition:

(**) at each point $x$, either $D^{+} f(x)=+\infty$ or $D_{+} f(x)=0$, and at each point $x$, either $D^{-} f(x)=\infty$ or $D_{-} f(x)=0$.

In this note we will construct a strictly increasing continuous function $f$ satisfying condition $(* *)$. Thus $f$ cannot have a positive real unilateral derivative at any point.

It is worth comparing $f$ with a nondifferentiable function $p$ constructed in [2]. At each point $x$ either $D^{+} p(x)\left(D^{-} p(x)\right)$ is as large as possible, $\infty$, or $D_{+} p(x)\left(D_{-} p(x)\right)$ is as small as possible, $-\infty$. For our continuous increasing function $f$, at each point $x$ either $D^{+} f(x)\left(D^{-} f(x)\right)$ is as large as possible, $\infty$, or $D_{+} f(x)\left(D_{-} f(x)\right)$ is as small as possible, 0 .

Key Words: Dini derivate, derivative, strictly increasing function.

Mathematical Reviews subject classification: 26A24, 26A48.

Received by the editors October 29,1999 
The construction of $f$ begins with the construction of two sequences of sets $\left(A_{n}\right)$ and $\left(B_{n}\right)$ such that each $A_{n}$ and $B_{n}$ is the union of finitely many compact intervals.

Among other things, $A_{n} \cup B_{n}$ will be [0,1], and $A_{n} \cap B_{n}$ will be a finite set. We will proceed by induction on $n$. Let $A_{1}=[0,1 / 2]$ and $B_{1}=[1 / 2,1]$. To form $A_{2}$ delete from each component $I$ of $A_{1}$ an open symmetric subinterval $J$ of $I$ such that

$$
2^{2}(\text { length } J)=(\text { length } I) .
$$

Make $B_{2}$ the closure of $[0,1] \backslash A_{2}$. To form $B_{3}$ delete from each component $I$ of $B_{2}$ an open symmetric subinterval $J$ of $I$ such that

$$
2^{3}(\text { length } J)=(\text { length } I) .
$$

Make $A_{3}$ the closure of $[0,1] \backslash B_{3}$. If $A_{1}, \ldots, A_{n-1}$ and $B_{1}, \ldots, B_{n-1}$ have been constructed and if $n$ is even, form $A_{n}$ by deleting from each component $I$ of $A_{n-1}$, the open symmetric subinterval $J$ of $I$ with

$$
2^{n}(\text { length } J)=(\text { length } I),
$$

and make $B_{n}$ the closure of $[0,1] \backslash A_{n}$. If $n$ is odd, form $B_{n}$ by deleting from each component $I$ of $B_{n-1}$ the open symmetric subinterval $J$ of $I$ such that

$$
2^{n}(\text { length } J)=(\text { length } I),
$$

and make $A_{n}$ the closure of $[0,1] \backslash B_{n}$. By inductive construction, $A_{n}$ and $B_{n}$ have been constructed for all indices $n$. Note that the lengths of the components of $A_{n}$ and $B_{n}$ tend to 0 as $n \rightarrow \infty$.

Put

$$
A=\cap_{k=1}^{\infty} \cup_{j=k}^{\infty} A_{j} \quad \text { and } \quad B=\cap_{k=1}^{\infty} \cup_{j=k}^{\infty} B_{j} .
$$

It follows that $A \cup B=[0,1]$. (The set $A \cap B$ is nonvoid, but that will not affect our argument.)

Lemma 1. Let $[a, b]$ be a component interval of $A_{n}$ and $a \leq x<b$. Let $m$ denote the Lebesgue measure. Then

$$
m([x, b] \cap B) \leq 2^{1-n}(b-x) \quad \text { and } \quad m([x, b] \cap A) \geq\left(1-2^{1-n}\right)(b-x) .
$$

Proof. Either $B_{n+1} \backslash B_{n}$ is void or $[a, b] \cap\left(B_{n+1} \backslash B_{n}\right)$ consists of one subinterval of $[a, b]$ depending on whether $n$ is even or odd. It follows from the construction that the length of this interval is not greater than $2^{-n}(b-x)$.

Now $b$ is the right endpoint of a component of $A_{n+k}$ for $k=1,2,3, \ldots$ Thus $[x, b] \cap A_{n+k}$ consists of finitely many components of $A_{n+k}$ and/or a 
compact interval containing $x$. Repeated applications of the principle in the preceding paragraph and $B_{n+k} \backslash B_{n+k-1} \subset A_{n+k-1}$ show that

$$
\begin{gathered}
m\left([x, b] \cap\left(B_{n+k} \backslash B_{n+k-1}\right)\right)=m\left([x, b] \cap\left(B_{n+k} \backslash B_{n+k-1}\right) \cap A_{n+k-1}\right) \\
\leq 2^{1-n-k} m\left([x, b] \cap A_{n+k-1}\right) \leq 2^{1-n-k}(b-x) .
\end{gathered}
$$

But $m\left(A_{n} \cap B_{n}\right)=0$, and it follows that

$$
\begin{aligned}
m\left([x, b] \cap\left(\cup_{k=1}^{\infty} B_{n+k}\right)\right) & =m\left([x, b] \cap\left(\cup_{k=1}^{\infty}\left(B_{n+k} \backslash B_{n+k-1}\right)\right)\right) \\
& \leq \sum_{k=1}^{\infty} 2^{1-n-k}(b-x)=2^{1-n}(b-x) .
\end{aligned}
$$

Consequently $m([x, b] \cap B) \leq 2^{1-n}(b-x)$. But $A \cup B=[0,1]$, so $m([x, b] \cap A) \geq$ $\left(1-2^{1-n}\right)(b-x)$. This proves Lemma 1 .

Let the function $h$ be the indefinite integral of the characteristic function of $A$. Now, if $x$ lies in $\left[a_{n}, b_{n}\right)$ for components $\left[a_{n}, b_{n}\right]$ of infinitely many sets $A_{n}$, then from

$$
m\left(\left[x, b_{n}\right] \cap A\right) /\left(b_{n}-x\right) \geq\left(1-2^{1-n}\right)
$$

it follows that $D^{+} h(x)=1$. By reversing the roles of the sets $A_{n}$ and $B_{n}$, we see that if $x$ lies in $\left[c_{n}, d_{n}\right)$ for components $\left[c_{n}, d_{n}\right]$ of infinitely many sets $B_{n}$, then from

$$
m\left(\left[x, d_{n}\right] \cap A\right) /\left(d_{n}-x\right) \leq 2^{1-n}
$$

it follows that $D_{+} h(x)=0$. Thus for $x \in[0,1)$, either $D^{+} h(x)=1$ or $D_{+} h(x)=0$. We reverse left and right to see that for $x \in(0,1]$, either $D_{-} h(x)=0$ or $D^{-} h(x)=1$.

Now any subinterval $I$ of $[0,1]$ contains component intervals of some $A_{n}$ and $B_{n}$, and from the inequalities in the preceding paragraph it follows that the functions $h(x)$ and $x-h(x)$ are strictly increasing.

Put $k=h^{-1}$ on the set $h(0,1)$. Then the function $k(y)-y$ is strictly increasing on $h(0,1)$ because $x-h(x)$ is strictly increasing on $(0,1)$. From $D^{+} h\left(x_{0}\right)=1$ we obtain $D_{+} k\left(y_{0}\right)=1$ where $y_{0}=h\left(x_{0}\right)$, and from $D_{+} h\left(x_{0}\right)=$ 0 we obtain $D^{+} k\left(y_{0}\right)=\infty$. Likewise from $D^{-} h\left(x_{0}\right)=1$ we obtain $D_{-} k\left(y_{0}\right)=$ 1 , and from $D_{-} h\left(x_{0}\right)=0$ we obtain $D^{-} k\left(y_{0}\right)=\infty$. At each point in $h(0,1)$, either $D^{+} k=\infty$ or $D_{+} k=1$, and either $D^{-} k=\infty$ or $D_{-} k=1$.

Finally, $f(y)=k(y)-y$ is a continuous strictly increasing function on $h(0,1)$ satisfying condition $(* *)$. 
We conclude with the observation that if all that is required is a strictly increasing continuous function whose derivative vanishes almost everywhere, one solution is well-known and easily constructed. It can be found in $[3, \mathrm{p}$. $101]$.

\section{References}

[1] E. Hewitt and K. Stromberg, Real and Abstract Analysis, Springer Verlag, New York, 1965.

[2] A. P. Morse, A continuous function with no unilateral derivatives, Trans. Amer. Math. Soc. 44 (1938), 496-507.

[3] S. Saks, Theory of the Integral, Dover, New York, 1964. 